\title{
La PET/TC per la diagnostica avanzata in endocrinologia
}

\author{
Ines Bucci ${ }^{1}$. Cesidio Giuliani ${ }^{1}$. Giorgio Napolitano ${ }^{1}$
}

Accettato: 12 novembre 2020 / Pubblicato online: 10 agosto 2021

(c) The Author(s) 2021

\section{Introduzione}

La Tomografia a emissione di positroni (PET) è un'indagine medico-nucleare che utilizza molecole biologiche marcate con atomi emittenti positroni (radiofarmaci) e consente la valutazione di metabolismo, perfusione ed espressione recettoriale dei tumori. L'integrazione con un tomografo computerizzato (PET/TC) consente di associare il dato morfologico a quello molecolare-funzionale.

Diversi radiofarmaci "marcano" caratteristiche metabolico/molecolari delle neoplasie endocrine e il loro uso è crescente in endocrinologia (Tabella 1) [1]. Il ${ }^{18}$ F-fluorodesossiglucosio ( ${ }^{18}$ F-FDG) esplora il metabolismo del glucosio che è aumentato nelle cellule neoplastiche e infiammatorie. La 6- $\left[{ }^{18} \mathrm{~F}\right]$-L-fluoro-L-3, 4-diidrossifenilalanina $\left({ }^{18} \mathrm{~F}\right.$ FDOPA) si accumula nelle cellule di origine neuroendocrina. La ${ }^{18} \mathrm{~F}$-fluorocolina ( ${ }^{18} \mathrm{~F}$-Colina) evidenzia l'aumento del metabolismo dei fosfolipidi di membrana in condizioni di aumentata proliferazione cellulare [1]. I [ ${ }^{68} \mathrm{Ga}$ DDOTApeptidi sono analoghi della somatostatina a struttura simile ( ${ }^{68} \mathrm{GaDOTA}-\mathrm{TOC},{ }^{68} \mathrm{Ga}$-DOTA-NOC, ${ }^{68} \mathrm{Ga}$-DOTA-TATE), marcati attraverso un chelante (DOTA) al Gallio-68. Esplorano l'espressione dei recettori della somatostatina (SSTR) $[1,2]$.

Proposto da Giorgio Napolitano.

\footnotetext{
I. Bucci

ibucci@unich.it

1 Dipartimento di Medicina e Scienze dell'Invecchiamento, CAST, Università "G. d'Annunzio" Chieti-Pescara, Chieti, Italia
}

\section{Impiego clinico-diagnostico in endocrinologia}

\section{Carcinomi dell'epitelio follicolare della tiroide}

L'aumentata captazione del glucosio è un marker di dedifferenziazione dei carcinomi differenziati della tiroide (CDT) e si associa alla perdita della capacità iodocaptante, La ${ }^{18}$ F-FDG PET/TC è indicata nei pazienti con CDT che mostrano valori elevati di Tireoglobulina (Tg) senza captazione alla scintigrafia con ${ }^{131} \mathrm{I}$ e con reperti patologici assenti, o non proporzionati ai livelli del marcatore, all'imaging tradizionale. La sua sensibilità, variabile dal 50 al 100\%, dipende dal volume tumorale, dal grado di de-differenziazione $\mathrm{e}$, in misura minore, dallo stimolo del TSH; è maggiore quando i valori di $\mathrm{Tg}$ superano $10 \mathrm{ng} / \mathrm{mL}$ [3]. Nei carcinomi scarsamente differenziati la ${ }^{18} \mathrm{~F}$-FDG PET/TC è indicata anche nella stadiazione e nella valutazione del rischio di progressione e della risposta alle terapie.

\section{Carcinoma midollare della tiroide}

I radiofarmaci metabolici ${ }^{18} \mathrm{~F}$-FDG e ${ }^{18} \mathrm{~F}$-FDOPA forniscono informazioni complementari nei pazienti con carcinoma midollare della tiroide (CMT) con residuo/recidiva di malattia e nella valutazione della risposta alle terapie. La detection rate della ${ }^{18} \mathrm{~F}$-FDG PET/CT varia dal 59 al $69 \%$; la sua sensibilità è positivamente correlata con i livelli di calcitonina (CT) e CEA e con il loro tempo di raddoppiamento ed è maggiore quando i loro valori superano 1000 $\mathrm{pg} / \mathrm{ml}$ e $5 \mathrm{ng} / \mathrm{ml}$, rispettivamente. La detection rate della ${ }^{18}$ F-FDOPA PET/CT è dell' $86 \%$ in pazienti con CT maggiore di $150 \mathrm{pg} / \mathrm{ml}$ e tempo di raddoppiamento inferiore a 1 anno (Fig. 1b,c) [4]. La captazione del ${ }^{18}$ F-FDG è espressione di un comportamento biologico aggressivo del 
Tabella 1 Principali radiofarmaci PET, razionale e impiego clinico in endocrinologia

\begin{tabular}{|c|c|c|c|}
\hline Radiofarmaco & Funzione & $\begin{array}{l}\text { Informazione } \\
\text { funzionale/molecolare }\end{array}$ & Impiego clinico \\
\hline $\begin{array}{l}{ }^{18} \mathrm{~F} \text {-fluorodesossiglucosio } \\
\left({ }^{18} \mathrm{~F} \text {-FDG }\right)\end{array}$ & $\begin{array}{l}\text { Analogo del glucosio, si } \\
\text { concentra, viene fosforilato } \\
\text { ma non entra nelle } \\
\text { successive tappe del } \\
\text { metabolismo glucidico } \\
\text { accumulandosi nella cellula }\end{array}$ & $\begin{array}{l}\uparrow \text { Attività glicolitica nelle } \\
\text { cellule neoplastiche e } \\
\text { infiammatorie }\end{array}$ & $\begin{array}{l}\text { Carcinomi tiroidei iodio-refrattari } \\
\text { Carcinomi tiroidei indifferenziati } \\
\text { Carcinoma midollare della tiroide } \\
\text { Feocromocitomi/paragangliomi metastatici } \\
\text { Tumori neuroendocrini aggressivi }\end{array}$ \\
\hline $\begin{array}{l}{ }^{18} \mathrm{~F} \text {-fluorocolina } \\
\left({ }^{18} \mathrm{~F}-\text { Colina }\right)\end{array}$ & $\begin{array}{l}\text { Viene fosforilata a lecitina } \\
\text { per incorporazione nella } \\
\text { membrana cellulare }\end{array}$ & $\begin{array}{l}\uparrow \text { Attività dell'enzima } \\
\text { colina-chinasi per aumentato } \\
\text { fabbisogno di fosfolipidi di } \\
\text { membrana nelle cellule } \\
\text { neoplastiche }\end{array}$ & Iperparatiroidismo primario \\
\hline $\begin{array}{l}\text { 6- }\left[{ }^{18} \mathrm{~F}\right]-\mathrm{L}-\text { fluoro-L-3, } \\
\text { 4-diidrossifenilalanina } \\
\left({ }^{18} \mathrm{~F} \text {-FDOPA }\right)\end{array}$ & $\begin{array}{l}\text { Trasportata dal trasportatore } \\
\text { di aminoacidi, decarbossilata } \\
a^{18} \text { F-dopamina e } \\
\text { immagazzinata in granuli di } \\
\text { stoccaggio }\end{array}$ & $\begin{array}{l}\uparrow \text { Captazione e sequestro } \\
\text { nelle cellule di derivazione } \\
\text { neuroendocrina }\end{array}$ & $\begin{array}{l}\text { Carcinoma midollare della tiroide } \\
\text { Feocromocitoma/paraganglioma } \\
\text { Tumori neuroendocrini }\end{array}$ \\
\hline$\left[{ }^{68} \mathrm{Ga}\right]$ DOTA-peptidi & Analoghi della somatostatina & $\begin{array}{l}\text { Espressione dei recettori per } \\
\text { la somatostatina }\end{array}$ & $\begin{array}{l}\text { Tumori neuroendocrini } \\
\text { Feocromocitomi/paragangliomi } \\
\text { Carcinoma midollare della tiroide }\end{array}$ \\
\hline
\end{tabular}
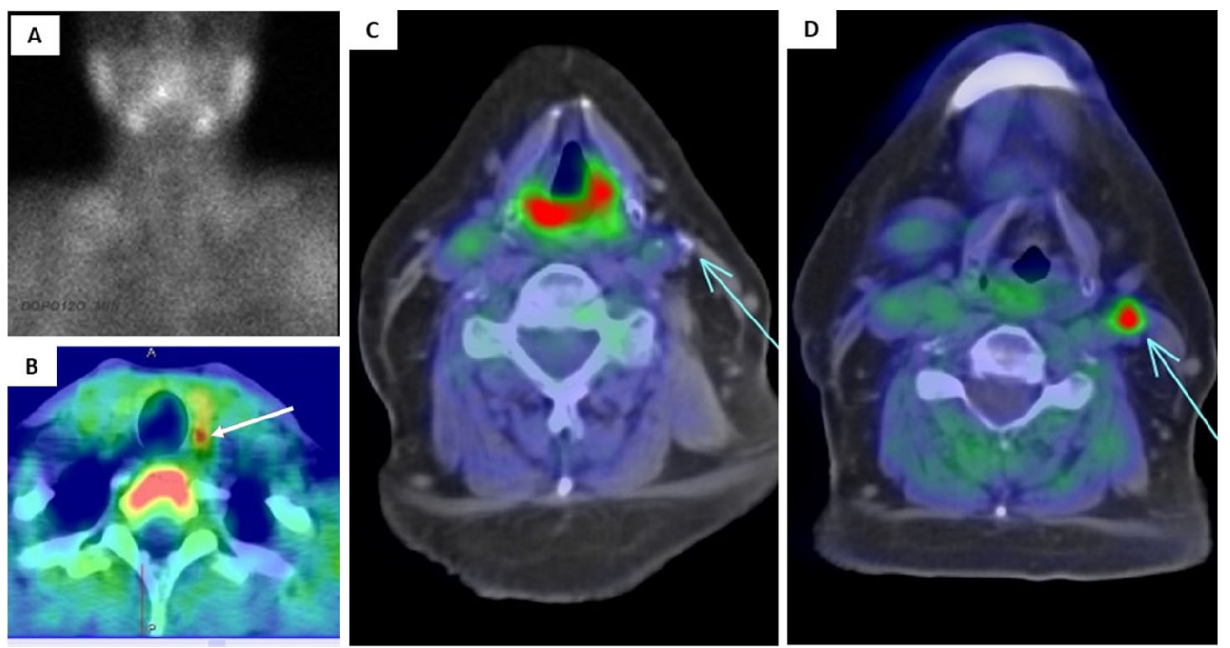

Fig. 1 a Scintigrafia ${ }^{99} \mathrm{mTc}$-Sestamibi con studio del wash-out del MI$\mathrm{BI}$, a $120 \mathrm{~min}$ non mostra tessuto paratiroideo iperfunzionante; $\mathbf{b}^{18} \mathrm{~F}$ FCH PET/TC, area di relativo iperaccumulo di ${ }^{18} \mathrm{~F}-\mathrm{FCH}$ in sede laterocervicale superiore di sinistra, posteriormente al III medio-inferiore del lobo tiroideo (paziente con iperparatiroidismo primario); ${ }^{18} \mathrm{~F}-\mathrm{FDG}$

tumore e si associa a una minore sopravvivenza. L'uso dei $\left[{ }^{68} \mathrm{Ga}\right] \mathrm{DOTA}$-peptidi è limitato dalla variabilità di espressione dei SSTR nel MTC ed è riservato a casi selezionati candidabili a terapia radio-recettoriale $[1,4]$.

\section{Iperparatiroidismo primario}

La ${ }^{18} \mathrm{~F}$-Colina, radiofarmaco di elezione per il carcinoma prostatico, è in grado di identificare paratiroidi iperplastiche. Nell'iperparatiroidismo primario la ${ }^{18} \mathrm{~F}$-Colina PET/TC ha
PET/CT non evidenza di aree di accumulo focale del tracciante; $\mathbf{d}{ }^{18} \mathrm{~F}$ FDOPA PET/CT focale accumulo del tracciante in sede laterocervicale sinistra (paziente operato per CMT, persistenza di malattia in sede linfonodale)

una sensibilità del 90\%, superiore a quella delle indagini tradizionali (ecografia del collo e scintigrafia ${ }^{99} \mathrm{mTc}$ SestaMIBI) (Fig. 1a,b). Può essere indicata in pazienti con imaging tradizionale negativo o inconclusivo [5].

\section{Feocromocitomi/paragangliomi}

Per il feocromocitoma il radiofarmaco di elezione è la ${ }^{18} \mathrm{~F}$ FDOPA, ha sensibilità e specificità elevate e il vantaggio di non richiedere preparazione e sospensione dei farmaci 
$[1,6]$. La ${ }^{18}$ F-FDOPA PET/TC ha indicazione elettiva anche nei paragangliomi (PG), specie a sede cervicale, mentre la ${ }^{18}$ F-FDG PET/TC è utile nei PG metastatici e nei pazienti con mutazioni del succinato deidrogenasi (SDHD). Emergente il ruolo della PET/TC con $\left[{ }^{68} \mathrm{Ga}\right] \mathrm{DOTA}$-peptidi che hanno mostrato una detection rate del 93\%, superiore alla ${ }^{18} \mathrm{~F}$-FDOPA e alla MIBI anche nelle lesioni piccole e in pazienti con mutazioni di SHDH [6]. Tali dati assumono rilevanza clinica nella selezione di pazienti candidabili alla terapia radio-recettoriale.

\section{Tumori neuroendocrini}

L'espressione dei SSTR nei tumori neuroendocrini (NET) a sede gastrointestinale e polmonare è il razionale dell'impiego dei $\left[{ }^{68} \mathrm{Ga}\right]$ DOTA-peptidi per: stadiazione, localizzazione del tumore primitivo in pazienti con metastasi accertate, selezione di pazienti candidati a terapia radio-recettoriale e $r e$ staging post-terapia. La sensibilità è superiore alle tecniche di imaging tradizionale [1]. Tutti i peptidi hanno buona affinità per i SSTR-2 (soprattutto il DOTA-TATE) mentre solo il DOTANOC ha affinità anche per i SSTR-3 e 5. Rispetto ai $\left[{ }^{68} \mathrm{Ga}\right]$ DOTA-peptidi la ${ }^{18} \mathrm{~F}$-FDOPA ha una sensibilità maggiore nei NET intestinali e minore nei pancreatici. La ${ }^{18} \mathrm{FDG}$ PET/TC ha una bassa sensibilità nei NET differenziati ed è indicata nei tumori più aggressivi.

Funding Note Open access funding provided by Università degli Studi G. D'Annunzio Chieti Pescara within the CRUI-CARE Agreement.

Conflitto di interesse Gli autori Ines Bucci, Cesidio Giuliani e Giorgio Napolitano dichiarano di non avere conflitti di interesse. Giorgio Napolitano fa parte del Comitato di redazione.

Consenso informato Lo studio presentato in questo articolo non ha richiesto sperimentazione umana.

Studi sugli animali Gli autori di questo articolo non hanno eseguito studi sugli animali.
Nota della casa editrice Springer Nature rimane neutrale in riguardo alle rivendicazioni giurisdizionali nelle mappe pubblicate e nelle affiliazioni istituzionali.

Open Access This article is licensed under a Creative Commons Attribution 4.0 International License, which permits use, sharing, adaptation, distribution and reproduction in any medium or format, as long as you give appropriate credit to the original author(s) and the source, provide a link to the Creative Commons licence, and indicate if changes were made. The images or other third party material in this article are included in the article's Creative Commons licence, unless indicated otherwise in a credit line to the material. If material is not included in the article's Creative Commons licence and your intended use is not permitted by statutory regulation or exceeds the permitted use, you will need to obtain permission directly from the copyright holder. To view a copy of this licence, visit http://creativecommons.org/licenses/by/4.0/.

\section{Bibliografia}

1. Bergeret S, Charbit J, Ansquer C et al (2019) Novel PET tracers: added value for endocrine disorders. Endocrine 64(1):14-30

2. Tirosh A, Kebebew E (2018) The utility of ${ }^{68}$ Ga-DOTATATE positron-emission tomography/computed tomography in the diagnosis, management, follow-up and prognosis of neuroendocrine tumors. Future Oncol 14:111-122

3. Haugen BR, Alexander EK, Bible KC et al (2016) 2015 American Thyroid Association management guidelines for adult patients with thyroid nodules and differentiated thyroid cancer: the American Thyroid Association Guidelines Task Force on Thyroid Nodules and Differentiated Thyroid Cancer. Thyroid 26:1-133

4. Giovanella L, Treglia G, Iakovou I et al (2020) EANM practice guideline for PET/CT imaging in medullary thyroid carcinoma. Eur J Nucl Med Mol Imaging 47:61-77

5. Evangelista L, Ravelli I, Magnani F et al (2020) 18F-choline PET/CT and PET/MRI in primary and recurrent hyperparathyroidism: a systematic review of the literature. Ann Nucl Med 34:601-619

6. Han S, Suh CH, Woo S et al (2019) Performance of 68Ga-DOTA conjugated somatostatin receptor targeting peptide PET in detection of pheochromocytoma and paraganglioma: a systematic review and meta-analysis. J Nucl Med 60(3):369-376 